\title{
Determination of stress fields and identification of thermomechanical parameters in a thermally grown oxide under thermal cycling loadings, using advanced models
}

\author{
Zhaojun Tao, , Felaniaina Rakotovao, ${ }^{2, \mathrm{~b}}$, Jean-Luc Grosseau-Poussard ${ }^{2, \mathrm{c}}$, \\ Benoit Panicaud ${ }^{1, d^{*}}$
}

${ }^{1}$ Université de Technologie de Troyes (UTT), Institut Charles Delaunay (ICD) - CNRS UMR 6279, Systèmes Mécaniques et d'Ingénierie Simultanée (LASMIS), 12 rue Marie Curie, CS 42060, 10004 Troyes Cedex, France

${ }^{2}$ Laboratoire des Sciences de l'Ingénieur pour l'Environnement (LaSIE) FRE-CNRS 3474, Université de La Rochelle, Avenue Michel Crépeau, 17042 La Rochelle Cedex 1, France

azhaojun.tao@utt.fr, 'belaniaina_nirisoa.rakotovao@univ-Ir.fr, jilgrouss@univ-Ir.fr, dbenoit.panicaud@utt.fr

Keywords: High temperature oxidation ; Stress measurements ; Optimization ; Thermal strain; Viscoplasticity ; Creep relaxation ; Residual stresses ; Chromium oxide ; Thermal cycling.

\begin{abstract}
The presence of residual stresses in thermal oxide layers has been recognized for a long time. In the present work, the mechanical fields for chromium oxide are investigated. An extended model is established to take into account the effects of temperature and thermal cycling for the calculation of oxide stress. Numerical results are given in order to predict the influence of different parameters, especially the dependence of some material parameters with temperature. This enables to make comparison with experimental results.
\end{abstract}

\section{Introduction.}

When metals or alloys are oxidized at high temperature, an oxide film generally develops. The existence of stress accompanying the oxide growth has been known for many years [1,2]. In particular, the role of stresses during the isothermal oxidation process may influence the structure and the protective properties of the oxide scales. Classical models (taking into account elasticity and/or viscoplasticity) are able to predict stress relaxation [1,3]. But, the origin of those stresses must, therefore, be sought-after in a growth strain related to the formation of the oxide above the substrate $[4,5]$.

Considering real loadings seen by the material (chromium oxide growing on $\mathrm{NiCr} 30$ alloy), it is important to take also into account the influence of temperature span and temperature rate during heating and cooling, in the modeling. Those factors have two kinds of influence, either a weak coupling through the dependence on temperature of the material parameters, or a strong coupling that links mainly the temperature rate to the thermal strain rate (thermal expansion).

In the present paper, the isothermal classical model is developed and upgraded through the use of the two previous couplings. In order to quantify the influence of these modifications, thermal cycling loadings are considered and applied using a numerical simulation. The temperature span and the temperature rate are particularly studied. Moreover, the choice of material parameters evolution with temperature is tested. The numerical results are eventually compared with experimental results found in literature.

\section{Modeling of the oxide stress evolution.}

Hypotheses. It is necessary to calculate the stresses evolution during the oxidation of a metal. The following assumptions are then made:

- The average stress is calculated taking into account the existence of a possible stress gradient.

- The force balance equation is considered in a quasi-static domain. 
- The system has an isotropic and biaxial behavior.

- The two-dimensional effects such as rumpling are not considered.

- The non-linear mechanical phenomena (buckling, cracking, spalling) are not considered.

- Therefore, there is displacement continuity at the oxide/metal interface.

- Only one single phased oxide layer is considered on the metal and this phase is timeindependent (no other chemical transformation are considered within the bulk of the oxide, except the oxidation itself). This "average oxide" has properties obtained by averaging oxides features.

- Chemical deformation due to dissolution of oxygen within the substrate alloy is not considered.

Growth strain. Huntz et al.[2] indicate several main possible origins for the growth strain during isothermal oxidation:

- the oxygen dissolution in the metal or alloy (incompatibility of the atomic volumes);

- the epitaxy (lack of compatibility of the crystalline lattices at interface);

- the Pilling and Bedworth approach (incompatibility of the molar volumes);

- the possibility that the oxide grows in the oxide grain boundaries.

These hypotheses are based on incompatibilities of some parameters leading to a stress state in the oxide layer. Depending on these hypotheses, there are several microstructural models. Recently, several authors have proposed that when the growth process occurs through the oxide grain boundaries, it induces a compressive lateral stress in the oxide film. A mixed flux is assumed (inward and outward diffusion) in the short circuits constituted of the oxide grain boundaries. It results an oxidation reaction along the grain boundaries in the layer. The oxide proportion located in the grain boundaries is assumed to be weak in comparison to the whole oxide layer, so that the kinetics remains controlled by the diffusion process in the short circuits. Thus, the oxide forms principally at the internal or external interfaces. But the weak oxide proportion that develops in the grain boundaries is sufficient to explain the appearance of stress in the oxide layer. Therefore, those microscopic strain incompatibilities induce a macroscopic lateral growth strain rate, perpendicularly to the oxide growth direction. The demonstration originally proposed by Clarke [4] and generalized by Panicaud et al. [5] indicates that the growth strain rate is proportional to the oxide layer kinetics, so that:

$$
\frac{d \varepsilon^{\text {growth }}}{d t}=D_{o x} \frac{d h_{o x}}{d t} .
$$

where $h_{o x}$ is the thickness of the oxide layer and $D_{o x}$ is a parameter related to some microstructural parameters (which is not a diffusion coefficient), which may depend on temperature. This approach is now well developed and has been investigated by several authors $[6,7,8]$.

Thermal strain. In the present paper, we are investigating effect of thermal cycling on the \{oxide + metal $\}$ system. It means that thermal strain through thermal expansion effect has to be taken into account, considering the following equation:

$$
\varepsilon^{\text {thermal }}=\alpha \times\left(T-T_{r e f}\right) \text {. }
$$

At the opposite of Eq. 1, this relation holds for both oxide and metal; $\alpha$ is the thermal expansion coefficient, which can depend on temperature.

Other strains. It is a priori necessary to distinguish two possible cases: symmetrical oxidation (when the two metallic sides are oxidized) and asymmetric oxidation (when the oxide forms on a single side). Thereafter, the oxidation will always be considered as symmetrical. The strain tensor in the layer is then uniform and depends only on time. On the other hand, the continuity equation can be separated during an isothermal oxidation in the following way [9]:

$$
\left(\varepsilon^{\text {elastic }}+\mathcal{E}^{\text {viscoplastic }}+\mathcal{E}^{\text {thermal }}+\mathcal{\varepsilon}^{\text {growth }}\right)_{o x}=\left(\mathcal{\varepsilon}^{\text {elastic }}+\mathcal{E}^{\text {viscoplastic }}+\mathcal{E}^{\text {thermal }}\right)_{m} .
$$

where $\varepsilon$ represents the lateral growth strain in the oxide, that would occur if no constraint were imposed. The classical Hooke law is used for the elastic strain, for an in-plane stress: 


$$
\mathcal{E}^{\text {elastic }}=\left(\frac{1-v}{E}\right) \times \sigma \text {. }
$$

$E$ is the Young modulus; $v$ is the Poisson ratio; both can depend on temperature.

A Norton-Hoff power law is used to simulate the viscoplastic strain in the metal and in the oxide for an in-plane stress [9], without hardening and without plastic threshold:

$$
\frac{d \varepsilon^{\text {viscoplastic }}}{d t}=\operatorname{sign}(\sigma) \times J \times|\sigma|^{N}=\operatorname{sign}(\sigma) \times \frac{1}{2}\left(\frac{|\sigma|}{K}\right)^{N} \text { and } N \in \mathrm{R} \text {. }
$$

where $J$ and $K$ are creep coefficients and $N$ is the Norton exponent, which can depend on temperature.

Equations of the modeling. To obtain the evolution of the stress within the oxide and the metal, it is necessary to consider a derivative form of the continuity relation (Eq. 3) that leads to Eq. 6:

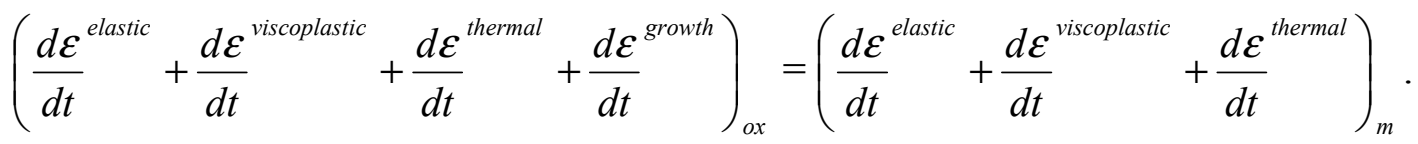

Therefore, behavior model for thermo-elasticity (Eqs. 2 and 4) have to be written in terms of rate. It gives:

$$
\frac{d \varepsilon}{d t}^{\text {thermal }}=\alpha(T) \frac{d T}{d t} .
$$

Derivative of Eq. 2 has to be done carefully, because it already and strictly corresponds to a differentiation. We have also:

$$
\frac{d \varepsilon}{d t}^{\text {elastic }}=\frac{d}{d T}\left(\frac{1-v}{E}\right) \frac{d T}{d t} \times \sigma+\left(\frac{1-v}{E}\right) \frac{d \sigma}{d t} .
$$

Eqs. 1 and 5 can be directly used. It is worth noting that Eq. 1 depends indirectly on temperature through a kinetic constant. For example, for parabolic evolution of the oxide thickness, we have:

$$
h_{o x}=A_{P} \sqrt{t} \Rightarrow \frac{d h_{o x}}{d t}=\frac{A_{P}}{2 \sqrt{t}}+\frac{d A_{P}}{d T} \frac{d T}{d t} \sqrt{t} .
$$

where $A_{P}$ is the parabolic kinetics constant. In addition, mechanical balance equation in the system has to be added:

$$
\int_{H_{0}}^{H_{1}} \sigma_{m} d z=-\int_{H_{1}}^{H_{2}} \sigma_{o x} d z .
$$

where the " $\mathrm{H}_{\mathrm{i}}$ " are the positions of the interfaces and $\mathrm{z}$ coordinate is defined by reference to a median line $\mathrm{H}_{0}$. The subscripts "ox" and " $\mathrm{m}$ " always refer respectively to oxide and metal.

\section{Influence of temperature on material parameters.}

Physical models. Because of temperature evolution, the parameters $J, K, N, E, v, \alpha, D_{o x}$ may vary. In the considered interval of temperature variation $\left(20^{\circ} \mathrm{C}\right.$ to $\left.900^{\circ} \mathrm{C}\right)$, some explicit value has to be given. The numerical values for some of the previous cited parameters for $\mathrm{Cr}_{2} \mathrm{O}_{3}$ on $\mathrm{NiCr} 30$ alloy can be found in $[1,2,10]$. With those values, different kind of fitting can be performed. First, we can consider a physical or phenomenological variation for each parameter with temperature. For example, it is classical to consider the evolution of elastic modulus such as [1]:

$$
E(T)=a_{0}-a_{1} T-a_{2} T^{2} .
$$

where the $a_{i}$ are constants. Moreover, the kinetics constant $A_{P}$ follows an Arrhenius dependence. Questions occur when considering the Norton coefficient $J$ and the growth parameter $D_{o x}$. Indeed, the viscoplasticity recovers several phenomena. For creep with diffusion of punctual defects, different mechanisms can occur either in grain (Nabarro-Herring) or in grain boundaries (Coble) [1]. Following such an explanation, the Norton coefficient is given by: 


$$
J(T)=\frac{\Omega C_{H N} D_{F i c k}^{G}(T)}{R T d^{2}(T)}\left(1+\frac{C_{C} D_{F i c k}^{G B}(T) \delta}{C_{H N} D_{F i c k}^{G}(T) d(T)}\right) .
$$

where $\Omega$ is the molar volume, $C_{H N}$ and $C_{C}$ are some constants, $D_{F i c k}$ are the diffusion coefficients in grain $(\mathrm{G})$ and in grain boundaries (GB), $R$ is the gas constant, $d$ is the grain size and $\delta$ is the grain boundary average thickness. This expression can be found in [1] and its validity has been verified in $[10]$ in the range of temperature $\left[700^{\circ} \mathrm{C}-900^{\circ} \mathrm{C}\right]$. By considering either one of the mechanism or the other (but not the mixed case) and if grain size and diffusion coefficient follow an Arrhenius model, we suggest the following expression, noted as model $\mathrm{J} 1$ (where effect of the microstructure is present in the exponential function):

$$
J(T)=\frac{\text { Cste }_{J 1}}{T} \exp \left(-Q_{J 1} / R T\right) .
$$

For the growth coefficient $D_{o x}$, it is more difficult, because mechanisms are not yet completely established. According to Clarke [4], the temperature dependence seems to be:

$$
D_{o x}(T)=\text { Cste }_{D} \exp \left(-Q_{1} / R T\right)+\text { Cste }_{D 2} \exp \left(-Q_{2} / R T\right) \text {. }
$$

If we consider the results found in [10], the authors suggest that the temperature dependence adapted to the present material may be:

$$
D_{o x}^{-1}(T)=D_{0}^{-1} \exp \left(-Q_{D 1} / R T\right) .
$$

This model is noted as D1.

Mathematical identification. For both creep and growth effects, other kinds of mechanism can be found (creep with dislocations...[1,2]). As a consequence, it is difficult to predict a general model with a universal dependence of the parameters with temperature. It is then always possible to consider the experimental results (for example those obtain in [10]) and try to fit with the best mathematical function. After some trials, the best fitting for Norton coefficient seems to be a power law, noted as model $\mathbf{J} 2$ :

$$
J(T)=C_{A} \times\left(T^{n}\right)+C_{B} .
$$

Whereas the growth parameter can be fitted using a sum of exponential, which is not the same as the model of Clarke of Eq. 14:

$$
D_{o x}(T)=C_{1} \exp \left(C_{2} \times T\right)+C_{3} \exp \left(C_{4} \times T\right) .
$$

This model is noted as D2, whose physical interpretation has yet to be investigated. Those models, given by Eqs. 16 and 17, are chosen here from a pure mathematical point of view.

\section{Numerical results in chromium oxide $\mathrm{Cr}_{2} \mathrm{O}_{3}$ growing on $\mathrm{NiCr30}$ alloy.}

The computation of the ordinary differential equations governing the stresses evolution, given by Eq. 1 and 5 to 10, has been performed with a Runge-Kutta numerical explicit scheme programmed in Matlab(C language. Details on numerical aspects can be found in [10]. The geometry is a onedimensional one. The considered thermal loadings are detailled in Figs 1. It corresponds to thermal cycles, for which either the temperature rate $\mathrm{T}_{-}$dot or the temperature span $\Delta \mathrm{T}$ is varying. The maximum temperature value is chosen to be $900^{\circ} \mathrm{C}$. The material parameters and their variation have been chosen according to isothermal experimental results [10] and compared to models such as those of Eqs. 11, 13, 15, 16 and 17. For both creep and growth parameters, the two models and set of parameters are compared (J1 vs. J2 and D1 vs. D2). The fitting of models parameters is shown in Figs. 2 and 3. Values of those parameters are given in Table 1. 
Table 1: Parameters corresponding to Eq. 13 (J1), Eq. 15 (D1), Eq. 16 (J2) and Eq. 17 (D2)

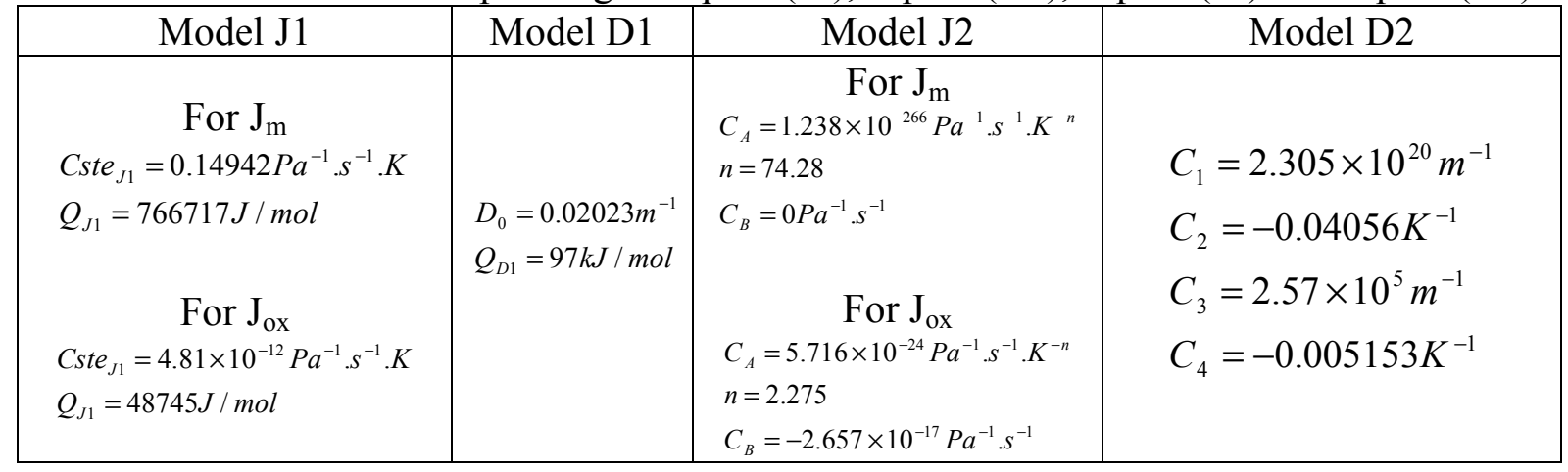

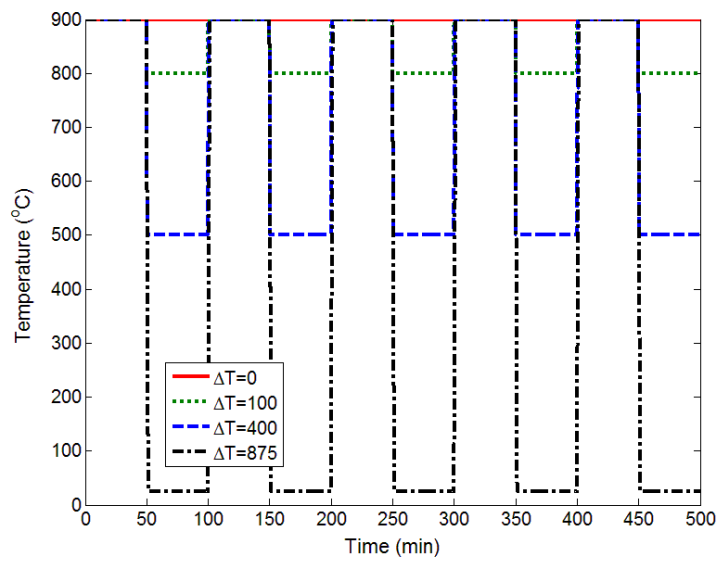

Figure 1 : Thermal loadings applied

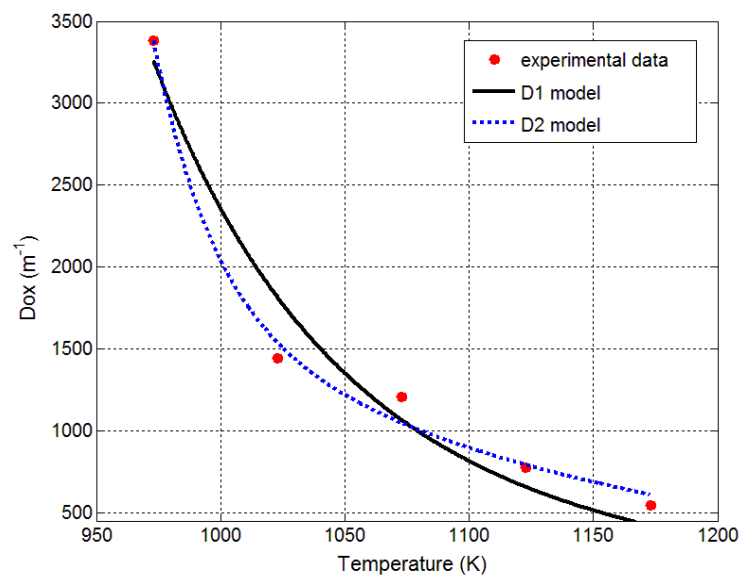

Figure 3 : Models for temperature evolution of the growth parameter $\mathrm{D}_{\mathrm{ox}}$

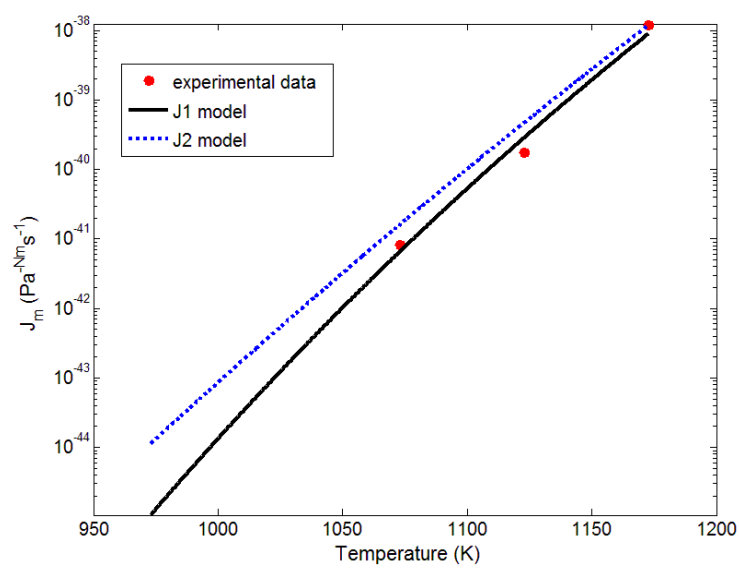

Figure 2 : Models for temperature evolution of the Norton coefficient $\mathrm{J}_{\mathrm{m}}(\log$ scale)

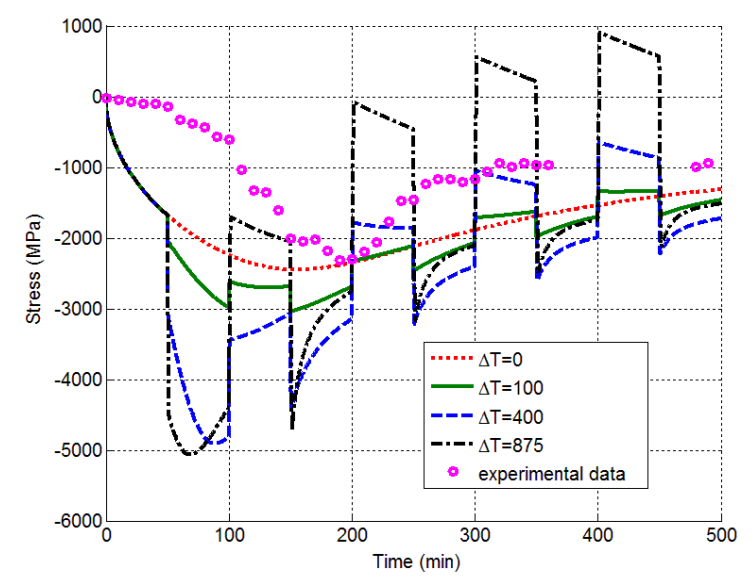

Figure 4 : Oxide stress versus oxidation time for different temperature spans, for a temperature rate of $600 \mathrm{~K} / \mathrm{min}$ and models $\mathrm{J} 2$ and D2

Numerical results are presented in Figs. 4 to 6 . The evolution of the oxide stress is presented as a function of the oxidation time. This evolution has been tested for different values of the temperature span $\Delta \mathrm{T}$ of $0,100,400,875 \mathrm{~K}$ (at a given temperature rate and a given model Fig. 4). The evolution has been also tested for different values of the temperature rate $\mathrm{T}$ dot of 10, 50, 100, 500 $\mathrm{K} / \mathrm{s}$ (at a given temperature span and a given model Fig. 5). Eventually, the different possible evolutions of material parameters with temperature are compared in Fig. 6. 


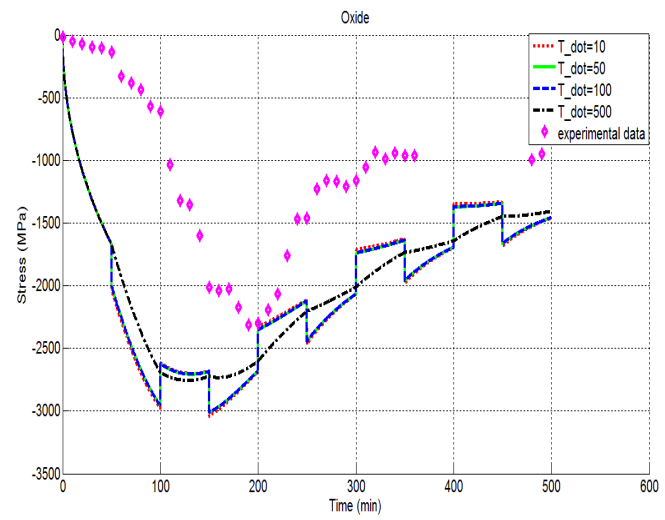

Figure 5 : Oxide stress versus oxidation time for different temperature rates, for a temperature span of $100 \mathrm{~K}$ and models $\mathrm{J} 2$ and D2

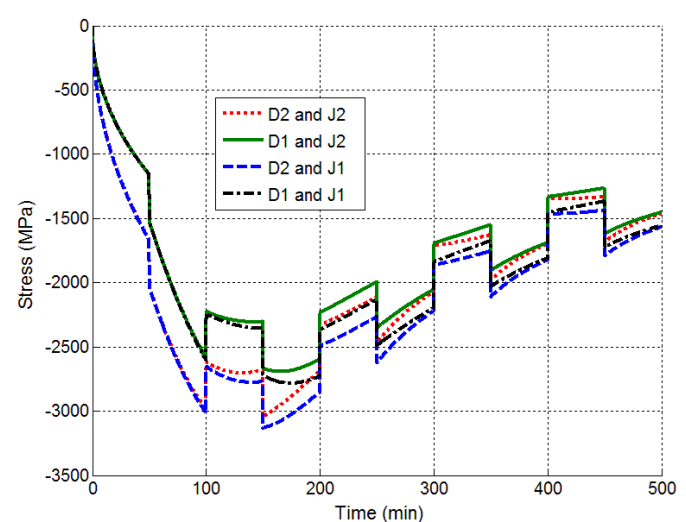

Figure 6 : Oxide stress versus oxidation time for different models, for a temperature rate of $10 \mathrm{~K} / \mathrm{s}$ and a temperature span of $100 \mathrm{~K}$

During the isothermal domain at $900^{\circ} \mathrm{C}$, the mechanical behavior of the model is in good agreement with the experimental results [10]. Small differences can be seen at short times for which the model remains to be improved. Moreover, the numerical results show that the mechanical response of the oxide follows the thermal loading with a characteristic time. An exponential behavior is found. This evolution agrees well with results found in [11]. Fig. 4 shows that the evolution of the stress is higher as the temperature span increases. Tensile stress may even appear in the oxide film as the relaxation takes place. Similarly, Fig. 5 shows that the evolution of the stress is smoother as the temperature rate increases. Besides, the different models used to fit the evolution of growth and creep parameters with temperature are not very sensitive.

\section{Conclusions.}

In the present work, it has been possible to propose an evolution of the mechanical model for a thermal oxide growing on a metallic alloy. The temperature have two kinds of influence, either a weak coupling through the dependence of temperature on the material parameters, or a strong coupling that links mainly the temperature rate to the thermal strain rate (thermal expansion). Sensitivity of different thermal loading parameters (temperature rate and temperature span) have been investigated. Moreover, the influence of the fitting has been checked. For the isothermal domain, numerical results agree with experimental results with a simple evolution: increase of the stress due to growth strain and decrease of the stress due to relaxation strain, mainly viscoplasticity. For non-isothermal domain with cycles, the stress evolution is more complex and depends strongly on the temperature span and on the temperature rate.

\section{References}

[1] M. Schütze, Protective Oxide Scales and Their Breakdown, Wiley, 1991.

[2] A.M. Huntz, B. Pieraggi, Oxydation des Matériaux Métalliques, Hermes Science, Paris, 2003.

[3] A.M. Huntz, G.C. Amiri, H.E. Evans, G. Cailletaud, Oxid. Met. 57 (2002) 499.

[4] D.R. Clarke, Acta Materialia 51 (2003) 1393-1407.

[5] B. Panicaud, J.L. Grosseau-Poussard, J.F. Dinhut, Comp. Mat. Sci. 42 (2008) 286-294.

[6] J.L. Grosseau-Poussard, B. Panicaud, S. Ben Afia, Comp. Mat. Sci. 71 (2013) 47-55.

[7] S. Maharjan, X. Zhang, Z. Wang, Oxid. Met. 77 (2012) 93-106.

[8] J.L. Ruan, Y. Pei, D. Fang, Acta Mech. 22312 (2012) 2597-2607.

[9] J.R. Vaunois, J.M. Dorvaux, P. Kanouté, J.L. Chaboche, Eur. J. of Mech. A/Solids 42 (2013) 402-421.

[10] B. Panicaud, J.L. Grosseau-Poussard, M. Kemdehoundja, J.F. Dinhut, Comp. Mat. Sci. 46 (2009) 42-48.

[11] B.W. Veal, A.P. Paulikas, P.Y. Hou, Nature Materials 5 (2006) 349-351. 